\title{
On the Cohomology of Relative Babach Algebras
}

\author{
Alaa Hassan Noreldeen Mohamed ${ }^{1}$ \\ ${ }^{1}$ Department of Mathematics, Faculty of Science, Aswan University, Aswa, Egypt. \\ Correspondence: Alaa Hassan Noreldeen, Dept. of Mathematics, Faculty of Science, Aswan University, Egypt. \\ E-mail: ala2222000@yahoo.com
}

Received: July 28, 2019

doi:10.5539/mas.v13n10p1
Accepted: September 1, 2019

Online Published: September 3, 2019

URL: https://doi.org/10.5539/mas.v13n10p1

\begin{abstract}
We study the relative cohomology theory of Banach algebra and give some important basic theorem of it. More, we give discuss about some of properties which we require it in our investigation. At long last, we ponder the dihedral cohomology aggregate as definitions and hypotheses and we characterize the Banach $S$-relative dihedral cohomology gathering and some theorems.
\end{abstract}

Mathematics Subject Classification: 16E40, 9D55.

Keywords: Dihedral, Homology, relative, Banach algebra

\section{Introduction}

The primary point in our search is considering the properties of Banach S-relative dihedral cohomology group, at that point we give the simplicial cohomology which examined in 1945 by Hochschild \& Mac Lane. What's more, the cyclic cohomology which contemplated by (Tsygan, 1983).

Dihedral homology group was examined by (Tsygan, 1983), and the dihedral (co)homology group of a variable based math with the involution and personality was presented by (Loday, 1987) and (Krasauskas, 1987).

In the second part, we presented the Hochschild, cyclic and dihedral cohomology group of Banach algebra with involution and identity and some of essential hypotheses on it. What's more, we connect between the Banach hochschild cohomology group, Banach cyclic cohomology group and Banach dihedral cohomology group.

In our examination, we characterize the Banach $S$-relative dihedral cohomology group of Banach algebra $A$ with involution which denoted by $H D^{n}[(A, S), X]$ since $S$ is closed subset of $A$ and the extention of the exact sequence,

$$
0 \rightarrow C D^{n}[(A, S), X] \rightarrow C D^{n}(A, X) \rightarrow C D^{n}(A / S, X) \rightarrow 0
$$

Since we utilize it to demonstrate the sequence which we could get it which is the connection between the Banach $S$-relative dihedral cohomology group $H D^{n}[(A, S), X]$, Banach dihedral cohomology group

${ }^{\alpha} H D^{n}(A, X)$ where $\alpha= \pm 1$ and the Banach cyclic cohomology group $H C^{n}(A, X)$ since $A$ is an involutive unity of Banach algebra. What's more, we characterize the trace map on the Banach $S$-relative dihedral cohomology group

$$
\operatorname{tr}: \mathcal{M}_{r}(M) \rightarrow M
$$

Furthermore, we got the Morita invariance which is the mapping between the components and its matrices and contains around two maps. The first, is known as the trace map which the map from the homologies of matrices into the homologies of components. Furthermore, the second map, is known as the inclusion map which is the map from the homologies of the components into the homologies of matrices with that both of two maps are inverse to each other.

Lastly, we ponder the Mayer-vietoris sequence of Banach $S$-relative dihedral cohomology group which is the sequence enable us to discover more data about the homology to group, since the Mayer-vietoris sequence is the exact sequence which related among the homology of spaces $A$ and $B$, intersection $A \cap B$ and union $A \cup B$. Also, we can process this homologies group effortlessly, and the Mayer-vietoris sequence causes us in demonstrates of some critical hypotheses.

Presently we will present some of essential definitions and illustrations which we require in our investigation. 
Definition (1.1): (Kreyszig E., 1978)

Consider the linear space $X$ and the field of scalar $K$, then the pair $(X, f)$ is the normal linear space which the norm $\|f\|$ satisfy that:

(1) $\|f\| \geq 0$,

(2) $\|f\|=0$ iff $f=0$,

(3) $\|c f\|=|c|\|f\| \quad \forall c \in K$,

(4) $\|f+g\| \leq\|f\|+\|g\|$.

Definition (1.2): (Kreyszig E., 1978)

Let $\left\{f_{n}\right\}_{n \in \mathbb{N}}$ is the sequence which elements are in the normal space $X$, then $\left\{f_{n}\right\}_{n \in \mathbb{N}}$ is:

1- Convergence to $f$ such that; $\forall \varepsilon>0, n \geq 0 \Rightarrow\left\|f-f_{n}\right\|<\varepsilon$.

2- Cauchy such that; $\forall \varepsilon>0, m, n>0 \Rightarrow\left\|f_{m}-f_{n}\right\|<\varepsilon$.

Definition (1.3): (Ramesh, G., 2013)

Give $X$ be a normed linear space, I fall Cauchy sequences in $X$ are convergent, then $X$ is complete. Since $X$ is complete normed linear space, then $X$ is called Banach space.

\section{Example (1.4):}

Let $\mathbb{R}^{n}$ is the Euclidean space and $x, y \in \mathbb{R}^{n}$ such that $x=\left(\alpha_{1}, \alpha_{2}, \cdots, \alpha_{n}\right)$ and $y=\left(\beta_{1}, \beta_{2}, \cdots, \beta_{n}\right)$ and the metric defined as

$$
d(x, y)=\sqrt{\left(\alpha_{1}-\beta_{1}\right)^{2}+\cdots+\left(\alpha_{n}-\beta_{n}\right)^{2}}
$$

If $\mathbb{R}^{n}$ is defined with the norm as

$$
\|x\|=\left(\sum_{i=1}^{n}\left|\alpha_{i}\right|^{2}\right)^{1 / 2}=\sqrt{\left|\alpha_{1}\right|^{2}+\cdots+\left|\alpha_{n}\right|^{2}}
$$

Then the space $\mathbb{R}^{n}$ is Banach space.

Definition (1.5): (Ramesh, G., 2013)

Let $Y$ is a subset of $X$ since $X$ is the Banach space and $Y$ is normed space, then $Y$ is subspace of $X$.

Theorem (1.6):(Kreyszig E., 1978)

Let $Y$ is subspace of the Banach space $X$ and is complete iff $Y$ is closed subset of $X$.

Definition (1.7): (Ramesh, G., 2013)

If $X$ is the vector space and $x, y \in X$ such that the inner product, $\langle x, y\rangle$ have:

I- $\langle x, x\rangle$ is real and $\langle x, x\rangle \geq 0$,

II- $\langle x, x\rangle=0$ iff $x=0$,

III- $\langle x, y\rangle=\overline{\langle x, y\rangle}$,

IV- $\left\langle a x_{1}+b x_{2}, y\right\rangle=a\left\langle x_{1}, y\right\rangle+b\left\langle x_{2}, y\right\rangle$.

Since the standard, $\|x\|=\langle x, x\rangle^{1 / 2}$, at that point the inner product is normed linear space.

The Cauchy-schwarz inequality is, $|\langle x, y\rangle| \leq\|x\|\|y\| \quad \forall x, y \in X$.

Finally, we can define the Hilbert space as the Banach space which the inner product determines the norm. i.e. the Hilbert space is the complete inner product.

Example (1.8):( Kreyszig E., 1978)

Let $\mathbb{C}^{n}$ be the space which elements are complex number and the metricdefined as if $x, y \in \mathbb{C}^{n}$ where $x=\left(\alpha_{1}, \alpha_{2}, \cdots, \alpha_{n}\right)$ and $y=\left(\beta_{1}, \beta_{2}, \cdots, \beta_{n}\right)$, then

$$
d(x, y)=\sqrt{\left|\alpha_{1}-\beta_{1}\right|^{2}+\cdots+\left|\alpha_{n}-\beta_{n}\right|^{2}}
$$

Then $\mathbb{C}^{n}$ is Hilbert space if $\mathbb{C}^{n}$ defined with inner product as

$$
\langle x, y\rangle=\alpha_{1} \overline{\beta_{1}}+\cdots+\alpha_{n} \overline{\beta_{n}}
$$

and with norm 


$$
\|x\|=\left(\alpha_{1} \overline{\alpha_{1}}+\cdots+\alpha_{n} \overline{\alpha_{n}}\right)^{1 / 2}=\left(\left|\alpha_{1}\right|^{2}+\cdots+\left|\alpha_{n}\right|^{2}\right)^{1 / 2}
$$

Definition (1.9): (Ramesh, G., 2013)

Let $A$ is the Banach algebra, $A$ is known as the Banach algebra with unity if $A$ has the unit $e$ such that for all $x \in A$ there exist an element $y \in A$ called the inverse of $x$ such that, $x y=y x=e$.

Definition (1.10): (Kreyszig E., 1978)

For a Banach algebra $A$. For all elements $a \in A$ there exist $a^{*} \in A$ such that $\left(a^{*}\right)^{*}=a$ is called the involution of

$A$ and $A$ is called the Banach algebra with involution.

Definition (1.11): (Williams, D. P., 2011)

Let the involution of a component $x \in X$ is, $x *$ such that satisfy that:

$$
\left(x^{*}\right)^{*}=x, \quad\left(x y^{*}\right)^{*}=y^{*} x^{*}, \quad(x+\lambda y)^{*}=x^{*}+\bar{\lambda} y^{*} .
$$

In the event that $X *$-algebra is the algebra $X$ with involution and the Banach *-algebra is the Banach algebra $X$ with involution, then we call the homomorphism $\varphi: X \rightarrow Y$ (which is the map between the $*$-algebras) the *-homomorphism such that, $\varphi\left(x^{*}\right)=\varphi(x)^{*} \quad \forall x \in X$.

Also, we can characterize the $C^{*}$-algebra as the Banach *-algebra if $\left\|x^{*} x\right\|=\|x\|^{2} \quad \forall x \in X$.

Definition (1.12): (Gouda, Y. Gh., Alaa, H. N., 2013)

Let $f$ and $g: C \rightarrow \grave{C}$ are pre-simplicial maps, we can define the pre-simplicial homotopy as the collection $h_{i}: C_{n} \rightarrow C_{n+1}$ s. $h . i=0, \ldots, n$ and satisfies that:

$$
\begin{array}{ccc}
d_{i} h_{i}=h_{i-1} d_{i} & \text { if } i<j, \\
d_{i} h_{i}=d_{i} h_{i-1} \quad \text { if } 0<i<n, i=j, j+1, \\
d_{i} h_{j}=h_{j} d_{i-1} & \text { if } i>j+1, \\
d_{0} h_{0}=f \quad \text { and } & d_{n+1} h_{n}=g .
\end{array}
$$

where $d_{i}: C_{n} \rightarrow C_{n-1}$ are the face maps.

Before we talk about the cohomology group, we define the notation which we use in our studying.

Assume that $A$ is Banach algebra, $B \subset A$ and closed. At that point $H C^{n}(A)$ methods the Banach cyclic cohomology group of $A, H C_{B}^{n}(A)$ is the $B$-relative cyclic cohomology group of $A, H D^{n}(A)$ be the Banach dihedral cohomology group of $A$ and $H D_{B}^{n}(A)$ is the $B$-relative dihedral cohomology group of $A$. id be an identity operator, $\widehat{\otimes}$ be the projective tensor product for Banach space.

And we write $H D^{n}(A)_{X}$ instead of $H D^{n}(A, X)$ since $H D^{n}(A, X)$ is the Banach dihedral cohomology of algebra $A$ with elements in $X$.

\section{Hochschild, Cyclic and Dihedral Cohomology Group of Banach algebra}

Here, we ponder simplicial, Cyclic and dihedral cohomology group of the Banach algebra as definition, theorems and the connection among them. We use (Gouda \& Alaa (2013)), (Alaa (2019)), (Loday, J. L., 1991)) and (Krasauskas, Lapen \& Solovev (1987)) in this part.

Definition (2.1): (Loday, J. L., 1991)

Assume that $A$ be Banach algebra and $X$ be the Banach $A$-bimodule. Then we can characterize the cochain complex $C^{n}(A, X)$ as,

$$
0 \rightarrow C^{0}(A, X) \stackrel{\delta^{0}}{\longrightarrow} \ldots \rightarrow C^{n}(A, X) \stackrel{\delta^{n}}{\longrightarrow} C^{n+1}(A, X) \rightarrow \ldots
$$

Since $C^{0}(A, X)=X$ and the coboundary $\delta^{n}: C^{n}(A, X) \rightarrow C^{n+1}(A, X)$ is characterized as

$$
\left(\delta^{n} f\right)\left(a_{1}, \ldots, a_{n+1}\right)=a_{1} f\left(a_{2}, \ldots, a_{n+1}\right)+\sum_{i=1}^{n}(-1)^{i} f\left(a_{1}, \ldots, a_{i} a_{i+1}, \ldots, a_{n+1}\right)+(-1)^{n+1} f\left(a_{1}, \ldots, a_{n}\right) a_{n+1}
$$

Also, the kernel $Z^{n}(A, X)$ of $\delta^{n}$ are known as the $n$-cocyclies and the image $B^{n}(A, X)$ of $\delta^{n-1}$ are known as the coboundaries. What's more, $\delta^{n+1} \circ \delta^{n}=0$. We call the cohomology of $C(A, X)$ the Banach cohmology group of $A$ which the coefficients are in $X$ and given by; 


$$
H^{n}(A, X)=Z^{n}(A, X) / B^{n}(A, X)
$$

Definition (2.2): (Lykova, Z. A, 1999)

Give $M_{*}$ be a Banach $A$-bimodule, then the Banach $A$-bimodule $M=\left(M_{*}\right)^{*}$ is called double. Allow $X$ to be a normed Banach cohomology $H^{1}(A, M)=\{0\}$, then $A$ is called amenable. Furthermore, if $H^{1}(A, X)=\{0\}$, then $A$ is called contractible

Definition (2.3): (Lykova, Z. A, 1999)

Give $S$ be a closed sub-algebra of $A$, then the closed subspace $C_{S}^{n}(A, X)$ of $C_{S}^{n}(A, X)$ with $\rho$ is the sequence,

$$
0 \rightarrow C_{S}^{0}(A, X) \stackrel{\delta^{0}}{\longrightarrow} \ldots \rightarrow C_{S}^{n}(A, X) \stackrel{\delta^{n}}{\longrightarrow} C_{S}^{n+1}(A, X) \rightarrow \ldots
$$

such that, $\forall \quad a_{1}, a_{2}, \ldots, a_{n} \in A, \quad s \in S, \quad 1 \leq i \leq n$ satisfy that,

$$
\begin{gathered}
\rho\left(s a_{1}, a_{2}, \ldots, a_{n}\right)=s \rho\left(a_{1}, a_{2}, \ldots, a_{n}\right), \\
\rho\left(a_{1}, a_{2}, \ldots, a_{i-1}, a_{i} s, a_{i+1}, \ldots, a_{n}\right)=\rho\left(a_{1}, \ldots, a_{i-1}, a_{i}, s a_{i+1}, a_{i+2}, \ldots, a_{n}\right)
\end{gathered}
$$

What's more, $\rho\left(a_{1}, a_{2}, \ldots, a_{n} s\right)=\rho\left(a_{1}, a_{2}, \ldots, a_{n}\right) s$.

$C_{S}^{n}(A, X)$ is called $S$-relative $n$-cochains. The kernel of $\delta^{n}$ in $C_{S}^{n}(A, X)$ is indicated by $Z_{S}^{n}(A, X)$ and called $S$-relative $n$-cocycles, and the $S$-relative co-boundaries is the image of $\delta^{n-1}: C_{S}^{n-1}(A, X) \rightarrow C_{S}^{n}(A, X)$ and signified by $B_{S}^{n}(A, X)$. And afterward the cohomolgy group of $C_{S}^{n}(A, X)$ is the Banach $S$-relative cohomology group of $C_{S}^{n}(A, X)$ and given by;

$$
H_{S}^{n}(A, X)=Z_{S}^{n}(A, X) / B_{S}^{n}(A, X)
$$

\section{Proposition (2.4):}

Assume that $A$ be the Banach algebra, $B$ is the amenable closed sub-algebra of $A$, and $M$ is the double $A$-bimodule for $n \geq 1$. Let $\rho \in C^{n}(A, M)$ s. $h .\left(\delta^{n} \rho\right)\left(a_{1}, \ldots, a_{n+1}\right)=0$ in the event that one of $a_{1}, \ldots, a_{n+1} \in B$. Then there exists $\xi \in C^{n-1}(A, M)$ such that if for any of $a_{1}, \ldots, a_{n} \in B$.

\section{Lemma (2.5):}

Assume that the Banach algebra, $B$ is the closed subalgebra of $A, \mathrm{X}$ is a Banach $A$-bimodule and $n \geq 1$. Let $\rho \in C^{n}(A, X) s . h .\left(\delta^{n} \rho\right)\left(a_{1}, \ldots, a_{n+1}\right)=0$ in the event that one of $a_{1}, \ldots, a_{n+1} \in B$ and $\rho\left(a_{1}, \ldots, a_{n}\right)=0$ on the off chance that one of $a_{1}, \ldots, a_{n} \in B$. Then, $\rho \in C_{B}^{n}(A, X)$.

Definition (2.6): (Alaa, H. N. \& Gouda,Y. Gh., 2009)

Let $A$ be the Banach algebra with unity since the coefficients in $A$-bimodule $X$, then we can characterize the Banach cyclic cohomology group of $A$ as the homology of the cocomplex $\operatorname{Tot}_{C} C^{* *}(A)$




as

$$
H C^{n}(A, X)=H^{n}\left(\operatorname{Tot} C C^{* *}(A, X)\right)
$$

Where $C C^{* *}(A, X)$ is the bicomplex of cochain with the vertical maps $b^{*}$ and $b^{* *}: C C^{\alpha \beta} \rightarrow C C^{\alpha \beta+1}$ and the horizontal maps $(1-t)^{*}$ and $N^{*}: C C^{\alpha \beta} \rightarrow C C^{\alpha+1 \beta}$.

Definition (2.7): (Alaa Hassan 2013)

Let $A$ is the Banach algebra with unity and $S$ is the closed subalgebra of $A$, then we can present the Banach S-relative cyclic cohomology $H C_{S}^{n}(A, X)$ of the cochain exact complex

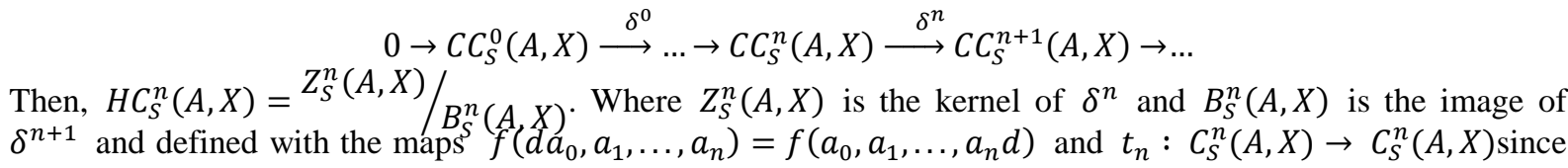
$\delta^{n+1}$ and defined with
satisfy the following;

$$
\begin{aligned}
t_{n} f\left(a_{0}, a_{1}, \ldots, a_{n}\right)= & (-1)^{n} f\left(a_{n}, a_{1}, \ldots, a_{0}\right) . \\
t^{n} f\left(d a_{0}, a_{1}, \ldots, a_{n}\right) & =(-1)^{n} f\left(a_{n}, a_{1}, \ldots, d a_{0}\right) \\
= & (-1)^{n} f\left(a_{1}, \ldots, a_{n} d, a_{0}\right)=t^{n} f\left(a_{0}, a_{1}, \ldots, a_{n} d\right) .
\end{aligned}
$$

Theorem (2.8):

Let $A$ be the Banach algebra with unity and $X$ the A-bimodule, then the connection between the Banach cyclic cohomology group and the Banach Hochschild cohomology group is the sequence,

Proof:

$$
\cdots \rightarrow H H^{n}(A, X) \stackrel{B}{\rightarrow} H C^{n-1}(A, X) \stackrel{S}{\rightarrow} H C^{n+1}(A, X) \stackrel{I}{\rightarrow} H H^{n+1}(A, X) \stackrel{B}{\rightarrow} \cdots
$$

Consider $C C(A, X)^{\{2\}}$ is the bicomplex such that contains the first and second columns of $C C(A, X)$ and $C[2,0]_{p q}=C_{p-2, q}$. Then the following sequence is exact

$$
0 \rightarrow C C(A, X)^{\{2\}} \rightarrow C C(A, X) \rightarrow C C(A, X)[2,0] \rightarrow 0
$$

The long exact sequence for this sequence is the sequence of cohomology of the Banach algebra since $C C(A, X)^{\{2\}}$ spoke to the Banach Hochschild cohomology group and $C C(A, X)$ be the Banach cyclic cohomology group. And then we get the required.

Theorem (2.9):

Let $S$ is the closed subalgebra of Banach algebra $A$ with unity and involution and $X$ is the A-bimodule, then we can get the connection between the Banach S-relative Cyclic cohomology group and the Banach S-relative Hochschild cohomology group as the sequence

$$
\cdots \rightarrow H H_{S}^{n}(A, X) \stackrel{B}{\rightarrow} H C_{S}^{n-1}(A, X) \stackrel{S}{\rightarrow} H C_{S}^{n+1}(A, X) \stackrel{I}{\rightarrow} H H_{S}^{n+1}(A, X) \stackrel{B}{\rightarrow} \cdots
$$

Definition (2.9): (Alaa Hassan Noreldeen, 2012)

Assume that $A$ be the Banach algebra with involution and $X$ be the Banach A-bimodule. Then we can characterize the Banach dihedral cohomology group $H D^{n}(A, X)$ of $A$ with coefficient in $X$ of the cochain complex

$$
0 \rightarrow C D^{0}(A, X) \stackrel{\delta^{0}}{\longrightarrow} \ldots \rightarrow C D^{n}(A, X) \stackrel{\delta^{n}}{\longrightarrow} C D^{n+1}(A, X) \rightarrow \ldots
$$

As the following sequence since we write $H D^{n}(A, X)$ instead of $H D^{n}(C(A, X))$

$$
\begin{aligned}
& 0 \rightarrow H D^{0}(A, X) \stackrel{\delta^{0}}{\longrightarrow} \ldots \rightarrow H D^{n}(A, X) \stackrel{\delta^{n}}{\longrightarrow} H D^{n+1}(A, X) \rightarrow \ldots \\
& \text { With the family of maps; } \quad \delta_{n}^{i}:[n-1] \rightarrow[n], \quad \sigma_{n}^{i}:[n+1] \rightarrow[n], \quad \tau_{n}:[n] \rightarrow[n]
\end{aligned}
$$
and $\rho_{n}:[n] \rightarrow[n]$ and satisfy the following: 


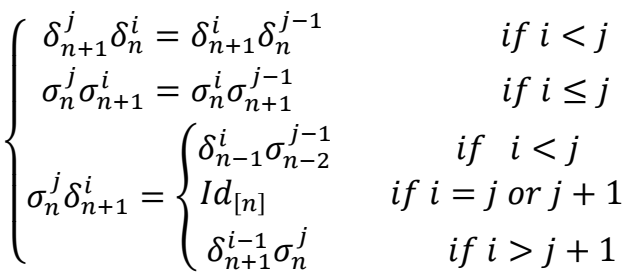

$$
\begin{aligned}
& \left\{\begin{array}{rlrl}
\tau_{n} \delta_{n}^{i}=\delta_{n}^{i-1} \tau_{n-1} & & 1 \leq i \leq n \\
\tau_{n} \sigma_{n}^{j}=\sigma_{n}^{j-1} \tau_{n+1} & 1 \leq j \leq n \\
\tau_{n}^{n+1}=I d_{[n]} &
\end{array}\right. \\
& \begin{cases}\rho_{n} \delta_{n}^{i}=\delta_{n}^{i-1} \rho_{n-1} & 0 \leq i \leq n \\
\rho_{n} \sigma_{n}^{j}=\sigma_{n}^{j-1} \rho_{n+1} & 0 \leq j \leq n\end{cases} \\
& \rho_{n}^{2}=I d_{[n]} \\
& \tau_{n} \rho_{n}=\rho_{n} \tau_{n}^{-1}
\end{aligned}
$$

$H D^{n}(A, X)$ is called the Banach dihedral cohomology group of $A$.

Theorem (2.10):

Let $A$ is the Banach algebra with unity and involution and coefficients in A-bimodule $X$. Then we can characterize the connection between the Banach cyclic cohomology group and the Banach dihedral cohomology group as the sequence,

$$
\cdots \rightarrow \quad-H D^{n}(A, X) \stackrel{i^{*}}{\rightarrow} H C^{n}(A, X) \stackrel{j^{*}}{\rightarrow} H D^{n}(A, X) \rightarrow \quad{ }^{-} H D^{n+1}(A, X) \rightarrow \cdots
$$

Proof:

Let $C(A, X)$ is the total complex of the bicomplex and $D(A, X)$ be the tricomplex. If $C(A, X)$ is embed in $D(A, X)$, then we get the short exact sequence,

$$
0 \rightarrow \operatorname{Tot} C(A, X) \rightarrow \operatorname{Tot} D(A, X) \rightarrow \operatorname{Tot} \quad-D(A, X) \rightarrow 0
$$

and we get,

$$
\cdots \rightarrow \quad-H D^{n}(A, X) \stackrel{i^{*}}{\rightarrow} H C^{n}(A, X) \stackrel{j^{*}}{\rightarrow} H D^{n}(A, X) \rightarrow \quad-H D^{n+1}(A, X) \rightarrow \cdots
$$

Theorem (2.11):

Let $A$ be the Banach algebra with unity and involution and coefficients in A-bimodule $X$. Then the connection among the Banach dihedral cohomology group, Banach cyclic cohomology group and Banach Hochschild cohomology group is the sequence,

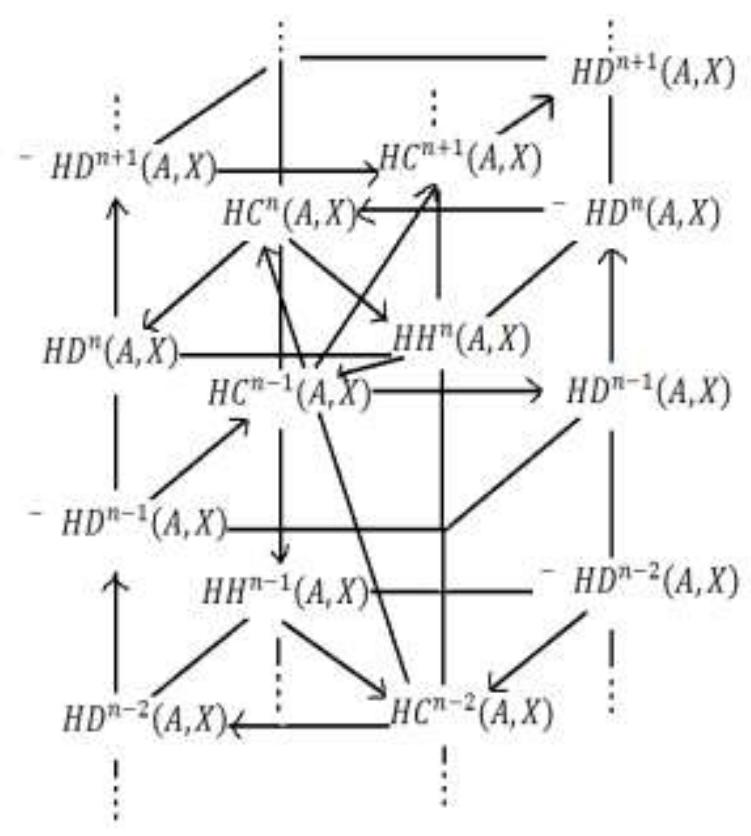




\section{Proof:}

From the theorem (4.2) which related between the dihedral cohomology group of Banach algebra and the cyclic cohomology group of Banach algebra and the theorem (3.2) which related between the Banach cyclic cohomology group and the Banach Hochschild cohomlgy group, then we get the required.

\section{Banach relative dihedral cohomology}

In this part, we defined the Banach relative dihedral cohomology and we study some of its properties and its relations with other cohomologies with it's proves. We use [(Alaa Hassan Noreldeen (2014),(2015))],[Gouda,Y. Gh., Alaa, H. N. \& M. Saad,(2017)], and [Gouda,Y. Gh., Alaa, H. N.,(2013) in this part.

\section{Definition (3.1):}

Consider $H D^{n}(A, X)$ be the Banach dihedral cohomology group of $A$ since $A$ is the Banach algebra with coefficients in $X$. Let $S$ be a closed subalgebra of $A$, then we can define the Banach $S$-relative dihedral cohomology group of Awhich denoted by $H D^{n}[(A, S), X]$ and we get the sequence of cohomology from the cochain exact complex;

$$
0 \rightarrow C D^{0}[(A, S), X] \stackrel{\delta^{0}}{\longrightarrow} \cdots \rightarrow C D^{n}[(A, S), X] \stackrel{\delta^{n}}{\longrightarrow} C D^{n+1}[(A, S), X] \rightarrow \cdots
$$

As the sequence,

$$
0 \rightarrow H D^{0}[(A, S), X] \stackrel{\delta^{0}}{\longrightarrow} \cdots \rightarrow H D^{n}[(A, S), X] \stackrel{\delta^{n}}{\longrightarrow} H D^{n+1}[(A, S), X] \rightarrow \cdots
$$

And the Banach S-relative dihedral cohomology group is given by,

$$
H D^{n}[(A, S), X]=Z^{n}[(A, S), X] / B^{n}[(A, S), X]
$$

Where, $Z^{n}[(A, S), X]$ is the kernel of $\delta^{n}$ and $B^{n}[(A, S), X]$ is the image of $\delta^{n+1}$ and satisfy the relation between the maps $\delta_{n}^{i}:[n-1] \rightarrow[n], \sigma_{n}^{i}:[n+1] \rightarrow[n], \tau^{n}:[n] \rightarrow[n]$ and $\rho^{n}:[n] \rightarrow[n]$ in definition (2.9).

Since the sequence which related between the Banach dihedral cochain complex $C^{n}(A, X)$ and the Banach S-relative dihedral cochain complex $C D^{n}[(A, S), X]$ is the sequence,

$$
0 \rightarrow C D^{n}[(A, S), X] \rightarrow C D^{n}(A, X) \rightarrow C D^{n}(A / S, X) \rightarrow 0
$$

And then we can get the relation between the Banach S-relative dihedral cohomology group is the sequence;

$$
\begin{gathered}
0 \rightarrow H D^{0}[(A, S), X] \rightarrow H D^{0}(A, X) \rightarrow H D^{0}(A / S, X) \rightarrow H D^{1}[(A, S), X] \rightarrow H D^{1}(A, X) \rightarrow \cdots \rightarrow H D^{n}[(A, S), X] \\
\rightarrow H D^{n}(A, X) \rightarrow H D^{n}(A / S, X) \rightarrow H D^{n+1}[(A, S), X] \rightarrow \cdots
\end{gathered}
$$

Theorem (3.2):

Let $S$ is the closed subalgebra of the Banach algebra $A$ with unity and involution and $X$ is the A-bimodule, then we can get the connection between the Banach S-relative Cyclic cohomology group and the Banach S-relative dihedral cohomology group as the sequence

$$
\cdots \rightarrow \quad-H D^{n}[(A, S), X] \stackrel{i^{*}}{\rightarrow} H C^{n}[(A, S), X] \stackrel{j^{*}}{\rightarrow} H D^{n}[(A, S), X] \rightarrow \quad{ }^{-} H D^{n+1}[(A, S), X] \rightarrow \cdots
$$

Proof:

Similar in theorem (2.10). 
Theorem (3.3):

Let $S$ is closed subset of the Banach algebra $A$ with involution and $X$ is the A-bimodule and let we write $H D^{n}(A, X)$ instead of ${ }^{\alpha} H D^{n}(A, X)$. Then the relation between the Banach relative dihedral cohomology group, Banach dihedral cohomology group and Banach cyclic cohomology group is the sequence

Proof:

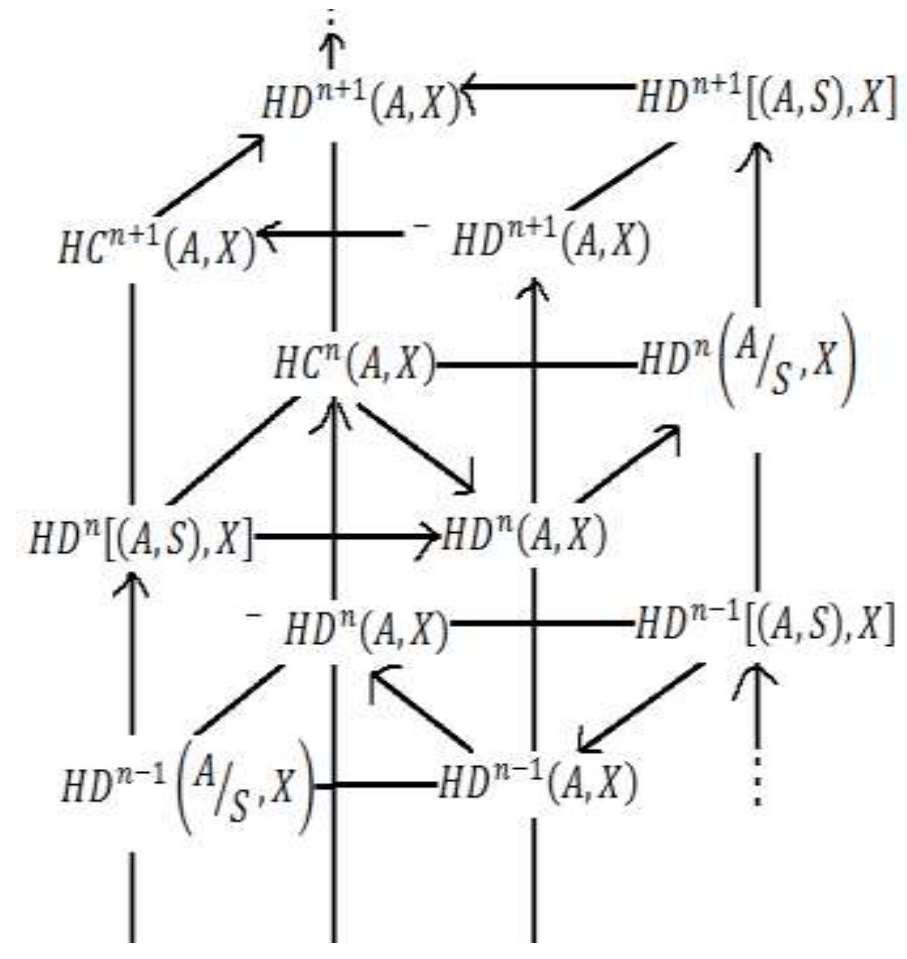

Let the following sequences are exact

$$
\begin{gathered}
0 \rightarrow H D^{0}[(A, S), X] \rightarrow H D^{0}(A, X) \rightarrow H D^{0}(A / S, X) \rightarrow H D^{1}[(A, S), X] \rightarrow H D^{1}(A, X) \rightarrow \cdots \rightarrow H D^{n}[(A, S), X] \\
\rightarrow H D^{n}(A, X) \rightarrow H D^{n}(A / S, X) \rightarrow H D^{n+1}[(A, S), X] \rightarrow \cdots
\end{gathered}
$$

And

$$
\cdots \rightarrow \quad-H D^{n}(A, X) \stackrel{i^{*}}{\rightarrow} H C^{n}(A, X) \stackrel{j^{*}}{\rightarrow} H D^{n}(A, X) \rightarrow \quad{ }^{-} H D^{n+1}(A, X) \rightarrow \cdots
$$

Then we get the required.

Definition (3.4):

Let $A$ is the Banach algebra with involution with coefficients in A-bimodule $\mathrm{X}, S$ is a subset of $A$ and $M$ is a bimodule which characterized over $S$ and $\mathcal{M}_{r}(M)$ is the matrices of degree $r \times r$. Then we can define the inclusion

$$
\text { inc: } \mathcal{M}_{r}(M) \rightarrow \mathcal{M}_{r+1}(M)
$$

As

$$
\alpha \mapsto\left[\begin{array}{cccc} 
& & & 0 \\
& \alpha & & \vdots \\
0 & \cdots & 0 & 0
\end{array}\right]
$$

Where $\operatorname{tr}: \mathcal{M}_{r}(M) \rightarrow M$ is the trace map and given by $\operatorname{tr}(\alpha)=\sum_{i=1}^{r} \alpha_{i i}$. And we define the generalized trace $\operatorname{map} \operatorname{tr}: \mathcal{M}_{r}(M) \otimes \mathcal{M}_{r}(S)^{\otimes n} \rightarrow M \otimes S^{\otimes n}$ as

$$
\operatorname{tr}(\alpha \otimes \beta \otimes \cdots \otimes \eta)=\sum\left(\alpha_{i_{0} i_{1}} \otimes \beta_{i_{1} i_{2}} \otimes \cdots \otimes \eta_{i_{n} i_{0}}\right)
$$

Theorem (3.5): (Morita invariance) 
Let $A_{X}$ is the Banach algebra with involution with coefficients in $X$ and $S_{X}$ is the subset of $A_{X}$, then for $r \geq 1$ the maps

$$
\operatorname{tr}^{*}: H D^{*}\left(\mathcal{M}_{r}\left(S_{X}\right), \mathcal{M}_{r}\left(M_{X}\right)\right) \rightarrow H D^{*}\left(S_{X}, M_{X}\right)
$$

and

$$
\operatorname{inc}^{*}: H D^{*}\left(S_{X}, M_{X}\right) \rightarrow H D^{*}\left(\mathcal{M}_{r}\left(S_{X}\right), \mathcal{M}_{r}\left(M_{X}\right)\right)
$$

are isomorphisms and both of them is inverse to each other.

Proof:

To demonstrate this theorem, we must show that (inc $\circ$ tr) and (id) are homotopic to each other. Let $h$ is the presimplicial homotopy since

$$
h=\sum(-1)^{i} h_{i} \text { and } \quad h_{i}: \mathcal{M}_{r}\left(M_{X}\right) \otimes \mathcal{M}_{r}\left(S_{X}\right)^{\otimes n} \rightarrow \mathcal{M}_{r}\left(M_{X}\right) \otimes \mathcal{M}_{r}\left(S_{X}\right)^{\otimes n+1}
$$

and defined by

$$
h_{i}\left(a^{0}, \ldots, a^{n}\right)=\sum E_{j 1}\left(a_{j k}^{0}\right) \otimes E_{11}\left(a_{k m}^{1}\right) \otimes \ldots \otimes E_{11}\left(a_{p q}^{i}\right) \otimes E_{1 q}(1) \otimes a^{i+1} \otimes \ldots \otimes a^{n}
$$

Since $a^{0} \in \mathcal{M}_{r}\left(M_{X}\right)$ and the others $a^{s} \in \mathcal{M}_{r}\left(S_{X}\right)$ and $h_{i}$ satisfy the first, second and third relation in definition (1.9). Since $h=\sum_{i=0}^{n}(-1)^{i} h_{i}$ and if $n=0, h(a)=E_{j 1}\left(a_{j k}\right) \otimes E_{1 k}(1)$.

If $n=1, h(a, b)=E_{j 1}\left(a_{j k}\right) \otimes E_{1 k}(1) \otimes b-E_{j 1}\left(a_{j k}\right) \otimes E_{11}\left(b_{k i}\right) E_{1 l}(1)$. Then, $h d+d h=d_{0} h_{0}-d_{n+1} h_{n}$. Since $i d=d_{0} h_{0}$ and $d_{n+1} h_{n}=i n c \circ t r$, then $i d$ and inc $\circ$ trare homotopic with each other.

Theorem (3.6):

Let $B$ is a closed Banach sub-algebra of the Banach $A$ with involution and coefficients in A-bimodule $X$ and $N \subset A, \quad K, J \subset B$ s. $h . \quad \cup K \subset A, \quad \cup J \subset B$. Then the Mayer-vietoris sequence of the Banach B-relative dihedral cohomology group is the sequence,

$$
\begin{aligned}
& \cdots \rightarrow H D^{n-1}(L \cap N, K \cap J)_{X} \stackrel{\partial_{*}}{\rightarrow} H D^{n}(A, B)_{X} \stackrel{k_{*}-l_{*}}{\longrightarrow} H D^{n}(L, K)_{X} \oplus H D^{n}(N, J)_{X} \\
& \stackrel{\left(i_{*} j_{*}\right)}{\longrightarrow} H D^{n}(L \cap N, K \cap J)_{X} \stackrel{\partial_{*}}{\rightarrow} H D^{n+1}(A, B)_{X} \rightarrow \cdots
\end{aligned}
$$

Where

$$
\begin{array}{cc}
i_{A}: L \hookrightarrow L \cap N, \quad i_{B}: K \hookrightarrow K \cap J, j_{A}: N \hookrightarrow L \cap N, j_{B}: J \hookrightarrow K \cap J, k_{A}: A \hookrightarrow L, k_{B}: B \hookrightarrow K, \\
l_{A}: A \hookrightarrow N \text { and } l_{B}: B \hookrightarrow J
\end{array}
$$

and $\oplus$ means the direct sum of the Banach algebra.

Proof:

Let the sequence,

$$
0 \rightarrow C D^{n}(A, B) \stackrel{g}{\rightarrow} C D^{n}(L, K) \oplus C D^{n}(N, J) \stackrel{f}{\rightarrow} C D^{n}(L \cap N, K \cap J) \rightarrow 0
$$

Is short exact sequence, since $f$ and $g$ are the epimomorphism and monomorphism, respectively. Where, $f=k-l, g=(i, j)$ and $f \circ g=0$.

Let $(L \cap N, K \cap J)(U) \in C D^{n}(A, B)$, then

$$
(f \circ g)(A, B)(U)=f((L, K)(U),(N, J)(U))=0
$$

Where, $((L, K)(U),(N, J)(U)) \in\left[C D^{n}(L, K) \oplus C D^{n}(N, J)\right]$. Then we can get from it into the long exact sequence

$$
\begin{gathered}
\cdots \rightarrow H D^{n-1}(L \cap N, K \cap J)_{X} \stackrel{\partial^{*}}{\rightarrow} H D^{n}(A, B)_{X} \stackrel{\stackrel{k^{*}-l^{*}}{\longrightarrow}}{N} H D^{n}(L, K)_{X} \oplus H D^{n}(N, J)_{X} \\
\stackrel{\left(i^{*}, j^{*}\right)}{\longrightarrow} H D^{n}(L \cap N, K \cap J)_{X} \stackrel{\partial^{*}}{\rightarrow} H D^{n+1}(A, B)_{X} \rightarrow \cdots
\end{gathered}
$$

\section{References}

Alaa Hassan Noreldeen Mohamed. (2019). On the Hochschild cohomology theory of Aœ-algebra, Scientific African 5 (2019) e00115. https://doi.org/10.1016/j.sciaf.2019.e00115

Alaa Hassan Noreldeen Mohamed. (2013). Some results on the dihedral homology of Banach algebras. Life Sci. J. 2013, 10(4), 1216-1220, ISSN:1097-8135. 
Alaa Hassan Noreldeen. (2015). On the dihedral homology of algebra. Ciencia e Tecnica Vitivinicola' journal, 30(3), 0254-0223.

Alaa Hassan Noreldeen. (2014). On the (co)homology with inner symmetry of schemes. Life Sic. J 2014, 11(12), 698-703.

Alaa Hassan Noreldeen. (2012). On the Homology Theory of Operator Algebras. International Journal of Mathematics and Mathematical Sciences, 2012(368527), 13.

Alaa, H. N. \& Gouda, Y. Gh. (2013). On the entire cyclic cohomology of Involutive Banach algebras. Pensee Journa, 75(12), 0031-4773. https://doi.org/10.1080/00927879408825166.

Alaa, H. N. \& Gouda, Y. Gh. (2009). On the dihedral (co)homology for schemes. International Electronic Journal of Algebra, 5(2009), 106-113. https://dergipark.org.tr/download/article-file/232888.

Gouda,Y. Gh. \& Alaa, H. N. (2013). On the simplicial cohomology theory of algebra. Life Sci. J. 2013, 10(3), 2639-2644.

Gouda, Y. Gh., Alaa, H. N. \& M. Saad. (2017). Reflexive and dihedral (co)homology of Z/2 Graded Algebras. International journal of Mathematics and statistics Invention (IJMSI), 5(1), 23-31. https://doi.org/10.1155/S0161171201000849

Krasauskas R. L., Lapen S. V. \& Solovev Yu. P. (1987). Dihedral homology and cohomology. Basic notions and constructions, Mat, Sb. 133(1987), 25-48.

Kreyszig, E. (1978). Introductory Functional Analysis with Applications, University of Windsor, New York, 1978.

Loday, J. L. (1991). Cyclic Homology, springer-verlage. New York, 1991.https://doi.org/10.1007/978-3-662-21739-9

Loday, J.-L. (1987). Homologies diedrale et quaternique. Dihedral and quaternionic homology, 66(2), 119-148 (French). https://doi.org/10.1016/0001-8708(87)90032-6

Tsygan, B. L. (1983). The homology of matrix Lie algebras over rings and the Hochschild homology. Uspekhi Mat. Nauk, 38(230), 217-218. https://doi.org/10.1070/RM1983v038n02ABEH003481

Lykova, Z. A. (1999). Relative Cohomology of Banach Algebra. J. Operator theory, 41(1999), 23-53.

Ramesh, G. (2013). Banach Algebra, India.

Williams, D. P. (2011). Lecture Notes on $C^{*}$-algebras, Dartmouth College.

\section{Copyrights}

Copyright for this article is retained by the author(s), with first publication rights granted to the journal.

This is an open-access article distributed under the terms and conditions of the Creative Commons Attribution license (http://creativecommons.org/licenses/by/4.0/). 